\title{
Consecuencias de la implementación de técnicas anticonceptivas sobre la terminación de vacas descarte
}

\author{
Kornuta, L.M. ${ }^{1}$; Flores, M.S. ${ }^{2}$; Yáñez, E.A. ${ }^{3}$; Fernández, J.A. ${ }^{4}$ \\ ${ }^{1}$ Becario de Pregrado, Univ.Nac.del Nordeste (UNNE), Corrientes, Argentina. ${ }^{2}$ Maestrando, Maestría en \\ Produc.Animal Subtropical, Fac. Cs. Vet. (UNNE). ${ }^{3}$ Profesor de Nutrición y Alimentación (FCV, UNNE). \\ ${ }^{4}$ Profesor de Nutrición Animal, Fac.Cs.Agrarias (UNNE). E-mail: kornuta.martin@gmail.com
}

\begin{abstract}
Resumen
Kornuta, L.M.; Flores, M.S.; Yáñez, E.A.; Fernández, J.A.: Consecuencias de la implementación de técnicas anticonceptivas sobre la terminación de vacas descarte. Rev. vet. 28 : 1, 31-36, 2017. Con el objeto de evaluar el aumento de peso vivo (APV) y la terminación en vacas de descarte, mediante la aplicación de métodos anticonceptivos cruentos e incruentos, se desarrolló un diseño en bloques completos al azar y tres tratamientos: control (C), Dutto (DT) y Diub (DB), con los bloques definidos por 48 vacas cruza Brangus (B1), y 30 vacas Hereford y Braford (B2). Los parámetros evaluados fueron: APV y terminación, utilizándose como fuente de variación: peso vivo (PV), condición corporal (CC) y espesor de grasa dorsal (GD). PV y $\mathrm{CC}$ se determinaron al inicio y cada 30 días, y GD al inicio y final del ensayo. El ANOVA no arrojó interacción bloques por tratamientos, ni tampoco hubo diferencias significativas entre tratamientos para APV y terminación, sin embargo al analizar por bloques, en B1 se observó un APV mayor en los lotes tratados (DB $67 \mathrm{~kg}$ y DT $62 \mathrm{~kg}$ ) que en el lote C (59 kg). En B2 el APV fue mayor para DT (92 kg), mientras que para los demás tratamientos los resultados fueron similares (DB $80 \mathrm{~kg}, \mathrm{C} 78 \mathrm{~kg}$ ). Luego se realizó un análisis de regresión lineal del APV, comparando las pendientes de cada ecuación con el objetivo de predecir qué pasaría si el período de evaluación del APV fuese más prolongado. El B1 presentó diferencias significativas entre los coeficientes de las pendientes de APV a favor de DT respecto a C, y DT vs DB. En B2 las diferencias de APV fueron entre C con DT y DB. Es importante desde el punto de vista del manejo del rodeo en un establecimiento de cría, la aplicación de estos métodos anticonceptivos donde haya presencia de toros y la expectativa de producción de esta categoría animal sea vender vacas gordas como descarte, evitando preñeces no deseadas.
\end{abstract}

Palabras clave: vacas viejas, DIUB, peso corporal, condición corporal, grasa dorsal.

\begin{abstract}
Kornuta, L.M.; Flores, M.S.; Yáñez, E.A.; Fernández, J.A.: Effects of contraception methods on termination of cull cows. Rev. vet. 28: 1, 31-36, 2017. In order to evaluate live weight gain (LWG) and termination in cull cows by means of the application of invasive and non invasive contraception methods, a completely randomized block design was used as follows: block 1: 48 half-breed Brangus cows; block 2: 30 Hereford and Braford cows using three treatments: control (C), Dutto (DT) and Diub (DB). The evaluated parameters were LWG and termination, using as live weight (LW) as the variation source, body condition (BC) and dorsal fat thickness (DFT). LW and BC were determined at the beginning and every 30 days and DFT at the beginning and end of the test. The ANOVA analysis did not show significant differences among treatments for LWG, termination, or interaction of blocks by treatments. However, in block 1 a higher LWG was observed in treated groups (DB $67 \mathrm{~kg}$, DT $62 \mathrm{~kg}$ ) compared to C $(59 \mathrm{~kg})$. In block 2 LWG was higher for DT $(92 \mathrm{~kg})$, while for the other treatments the results were similar (DB $80 \mathrm{~kg}, \mathrm{C} 78 \mathrm{~kg}$ ). A linear regression analysis was performed to evaluate the behavior of the regression's slopes in order to predict what would happen in the case of a longer evaluation period. Block 1 showed differences in the slopes between the coefficients for DT compared to C, and DT vs DB. In block 2 there were differences between $\mathrm{C}$ with DT and DB. These contraceptive methods are important from the management point of view in breeding farms where bulls are present in order to avoid unwanted pregnancies.
\end{abstract}

Key words: old cows, DIUB, liveweight, body condition score, dorsal fat. 


\section{INTRODUCCIÓN}

Las vacas descarte representan una categoría del sistema productivo, las cuales deben ser eliminadas de éste por no contar con las características que le permitan ser eficientes en el rodeo de cría. Las causas que llevan al descarte pueden ser: problemas reproductivos, locomotores, oculares, conformacionales, patologías de ubre o senilidad, en este caso, vacas viejas cuyo estado dentario compromete su alimentación conduciendo a la reducción de su condición corporal (CC), causas que afectan su eficiencia reproductiva.

Para que el sistema sea más rentable es necesario evitar que estas vacas se preñen nuevamente, engordarlas y venderlas para ser faenadas. Por tal motivo, en estos vientres bovinos de descarte, existe la necesidad de implementar técnicas capaces de evitar las preñeces no deseadas y mejorar la eficiencia de los sistemas de engorde.

Con este objetivo surgieron métodos tales como la ovariotomía por la técnica de Dutto ${ }^{4}$ y la aplicación de anticonceptivos (dispositivos intrauterinos bovinos: Diub) ${ }^{12}$. Por medio de la castración quirúrgica se lograría suprimir el celo, induciendo a un aumento del peso vivo (PV) y también disminuiría el estrógeno plasmático, reduciendo la movilización de lípidos con la consiguiente amplificación del depósito de los mismos y el aumento de la CC.

Por el contrario, el Diub produce aumento del nivel de testosterona llevando a un estado de anovulación, permitiendo de esta manera la anticoncepción incruenta con una efectividad del $99 \%$. El aumento en la cantidad de andrógenos también genera un incremento de la masa muscular y mejora la redistribución de los depósitos grasos, de tal manera que el cambio hormonal que se provoca en la hembra genera la incorporación de rasgos físicos propios del macho ${ }^{12}$.

En conjunto, ambas técnicas actuarían impidiendo que las vacas descartadas se preñen nuevamente, y a su vez facilitando el manejo y permitiendo su adecuada terminación para la venta. Basado en tales premisas, el presente trabajo tuvo como objetivo evaluar cómo influye la aplicación de métodos anticonceptivos cruentos e incruentos, sobre el aumento de peso vivo y la terminación en vacas de descarte.

\section{MATERIAL Y MÉTODOS}

El trabajo se desarrolló en el establecimiento Yatay Corá (bloque 1) ubicado a $50 \mathrm{~km}$ de la ciudad de Chavarría, Corrientes, donde se utilizaron 48 vacas Brangus. Se encuentra en la zona de "lomadas arenosas, planicies y depresiones", con clima subtropical sin estación seca, promedios pluviales de $1300 \mathrm{~mm} /$ año y temperatura de $21^{\circ} \mathrm{C}^{2}$. El pastizal natural se caracterizó por la existencia de pastos cortos como Paspalum $s p$ (pasto horqueta), Sporobolus $s p$ y Desmodium $s p$ (pega pega) y pastos altos como Andropogon sp (paja colorada).

Por otra parte, en las instalaciones del INTA ubicadas en la localidad de Sombrerito, Corrientes (bloque
2), se utilizaron 30 vacas Hereford y Braford. El predio se encuentra en la zona de "albardones y planicies del Paraná", con clima subtropical sin estación seca, promedios/año de $1300 \mathrm{~mm}$ de precipitaciones y $22^{\circ} \mathrm{C}$ de temperatura ${ }^{2}$. El pastizal natural se compone de pastos cortos (Paspalum sp) y altos (Sorgastrum $s p$ ).

Todas las vacas incluidas en el ensayo, identificadas con caravanas numeradas, fueron diagnosticadas vacías y clínicamente sanas, destetadas, descartadas de la reproducción, seleccionadas según $C C$ entre 2 y 4,5 (escala 1-9) ${ }^{11}$ y por dentición insuficiente, considerando como tal medio diente o inferior, sin llegar a un desgaste excesivo que comprometa la cosecha del pasto.

Cada animal se consideró una unidad experimental, donde se evaluaron los siguientes parámetros: aumento de peso vivo (APV) y terminación, utilizando como fuentes de variación: PV; CC y espesor de grasa dorsal (GD/mm). Para la determinación de PV se utilizó una balanza mecánica con capacidad para $5.000 \mathrm{~kg}$. La CC fue estimada utilizando la escala del 1-9 ${ }^{11}$. La GD se determinó entre la $12^{\circ}$ y $13^{\circ}$ costilla, transversalmente a la columna vertebral, a 1/3 de distancia del límite medial del músculo Longissimus dorsi. Se realizó con un ecógrafo Falco (Pie Medical) munido de un transductor de $17 \mathrm{~cm}$ y 3,5 Mhz, utilizando el software respectivo.

El día 0 del ensayo se determinaron PV, CC y GD. El día 7 se aplicaron los tratamientos, debido a la contraindicación de realizar la castración quirúrgica el mismo día de la palpación rectal, y luego cada 30 días se determinaron CC y PV hasta finalizar el experimento. GD se determinó el día 0 y al finalizar el experimento.

El lote se dividió en tres grupos homogéneos de 16 y 10 vacas para los bloques 1 y 2 , respectivamente, que se asignaron aleatoriamente a los siguientes tratamientos: A: Dutto: castración quirúrgica por el método descripto por su creador ${ }^{4}$. B: Diub: aplicándose los dispositivos de acuerdo a la técnica descripta ${ }^{12}$, colocándose uno en cada cuerno, utilizando el Diub ${ }^{\circledR}$ B3. C: Control: No se aplicó ningún método anticonceptivo. El experimento se desarrolló entre el 15/09/2009 y el 18/12/2009 en el bloque 1 (94 días), y entre el 10/12/2009 y el 08/03/2010 en el bloque 2 ( 88 días).

Se utilizó un diseño experimental en bloques completos al azar, considerando como bloques a los establecimientos. Los resultados obtenidos fueron analizados estadísticamente con ANOVA y comparación de medias a través del test de Duncan, con $p<0,05$. Luego se realizó un análisis de regresión lineal, con el objetivo de evaluar el comportamiento de las pendientes de las distintas variables estudiadas en los tres tratamientos, en cada bloque, mediante el uso de variables ficticias (“dummy"), utilizando en ambos casos el software estadístico Infostat ${ }^{6}$.

\section{RESULTADOS Y DISCUSIÓN}

Durante el ensayo, en el bloque 1 se produjo disminución del número de animales (2 en los tratamientos Diub y Dutto y uno en el control), debido a causas cli- 
Tabla 1. Valores iniciales de PV, CC y GD según bloques y tratamientos $(\overline{\mathrm{x}} \pm \mathrm{DE})$.

\begin{tabular}{lcccccc}
\hline \multirow{2}{*}{ tratamiento } & \multicolumn{3}{c}{ bloque 1} & \multicolumn{3}{c}{ bloque 2} \\
\cline { 2 - 7 } & PV & CC & GD & PV & CC & GD \\
\hline control & $334,6 \pm 31 \mathrm{a}$ & $3,7 \pm 0,7 \mathrm{~b}$ & $3,3 \pm 0,7 \mathrm{~b}$ & $391,7 \pm 35 \mathrm{~b}$ & $3,1 \pm 0,7 \mathrm{a}$ & $2,7 \pm 0,6 \mathrm{a}$ \\
Diub & $319,6 \pm 46 \mathrm{a}$ & $3,8 \pm 0,7 \mathrm{~b}$ & $3,8 \pm 1,6 \mathrm{~b}$ & $390,2 \pm 43 \mathrm{~b}$ & $3,3 \pm 0,7 \mathrm{a}$ & $2,7 \pm 0,6 \mathrm{a}$ \\
Dutto & $331,8 \pm 35 \mathrm{a}$ & $3,6 \pm 0,8 \mathrm{~b}$ & $3,3 \pm 0,9 \mathrm{~b}$ & $390,5 \pm 22 \mathrm{~b}$ & $3,4 \pm 0,6 \mathrm{a}$ & $2,5 \pm 0,6 \mathrm{a}$ \\
\hline
\end{tabular}

Superíndices distintos en las líneas para las mismas variables indican diferencias significativas $(\mathrm{p}<0,05)$ entre bloques.

Tabla 2. Diferencias entre valores iniciales y finales de PV, CC y GD, en ambos bloques ( $\overline{\mathrm{x}} \pm \mathrm{DE})$.

\begin{tabular}{lcccccc}
\hline \multirow{2}{*}{ tratamiento } & \multicolumn{3}{c}{ bloque 1} & \multicolumn{2}{c}{ bloque 2} \\
\cline { 2 - 7 } & PV & CC & GD & PV & CC & GD \\
\hline control & $59 \pm 21 \mathrm{a}$ & $1,4 \pm 0,4 \mathrm{a}$ & $0,62 \pm 0,6 \mathrm{a}$ & $80 \pm 14 \mathrm{~b}$ & $2,3 \pm 0.9 \mathrm{~b}$ & $0,80 \pm 0,8 \mathrm{~b}$ \\
Diub & $67 \pm 17 \mathrm{a}$ & $1,8 \pm 0.7 \mathrm{a}$ & $0,30 \pm 1,4 \mathrm{a}$ & $81 \pm 16 \mathrm{~b}$ & $2,4 \pm 0.8 \mathrm{~b}$ & $0,86 \pm 1,0 \mathrm{~b}$ \\
Dutto & $62 \pm 16 \mathrm{a}$ & $1,6 \pm 0.6 \mathrm{a}$ & $0,57 \pm 1,0 \mathrm{a}$ & $91 \pm 17 \mathrm{~b}$ & $2,7 \pm 1,0 \mathrm{~b}$ & $1,21 \pm 0.7 \mathrm{~b}$ \\
\hline
\end{tabular}

Superíndices distintos en las líneas para las mismas variables indican diferencias significativas $(\mathrm{p}<0,05)$ entre bloques.

máticas, enfermedad infecciosa y proceso inflamatorio, que llevaron a disminuciones de CC y PV, y muerte en un caso. En la Tabla 1 se presentan los promedios de las variables determinadas al inicio del ensayo, y en la Tabla 2 las diferencias entre los valores iniciales y finales del ensayo, expresadas en promedio $(\overline{\mathrm{x}}) \pm$ desvío estándar (DE).

Se puede observar que los animales del bloque 2 fueron de mayor tamaño (mayor PV), aunque presentaron menor CC que los del bloque 1. No se observaron diferencias estadísticas para ninguna de las variables entre los tratamientos, dentro de cada bloque.

No se observaron interacciones bloques por tratamientos, ni efectos significativos del tratamiento, para ninguno de los parámetros evaluados. Analizando el APV surge que no hubo diferencias estadísticamente significativas entre tratamientos; de todos modos en el bloque 1 puede observarse un APV mayor en los lotes tratados que en el control. De igual manera en el bloque 2, se observó un APV mayor en los animales intervenidos por medio de Dutto, mientras que para los demás tratamientos Diub y control los resultados fueron similares. En cuanto a la terminación de los animales tampoco se presentaron diferencias significativas en las fuentes de variación estudiadas (Tabla 2).

Una de las causas atribuidas a estos resultados no esperados, es que el período de duración del ensayo fue solo de tres meses. Si bien es un período razonable de tiempo para evaluar ganancia de peso y terminación en bovinos, es posible que no fuera suficiente para obtener las diferencias esperadas. Por otra parte este período no pudo ser extendido, pues los animales alcanzaron el grado de terminación adecuado y fueron enviados a faena.
Ante esta situación, se consideró importante realizar un análisis de regresión lineal, donde se buscó evaluar el comportamiento de las pendientes, con el objetivo de predecir lo que pasaría si el período de evaluación fuese más prolongado.

Para el bloque 1 (Figura 1) se presentaron diferencias en las pendientes entre los coeficientes comparando al grupo control con Dutto $(\alpha=0,1)$, no así comparando al control con Diub y Dutto vs Diub. Las ecuaciones de regresión representadas en las líneas del gráfico fueron: para DIUB $y=0,7677 x-30445$; para DUTTO $y=0,7027 x-27829$ y para Control $y=0,6225 x-24606$.

Para el bloque 2 (Figura 2) también se presentaron diferencias entre los coeficientes cuando se comparó al control con Dutto $(\alpha=0,1)$, y al control con Diub $(\alpha=$ $0.05)$. Las ecuaciones de regresión representadas en las líneas del gráfico fueron: para DIUB $y=1,0242 x-40733$;

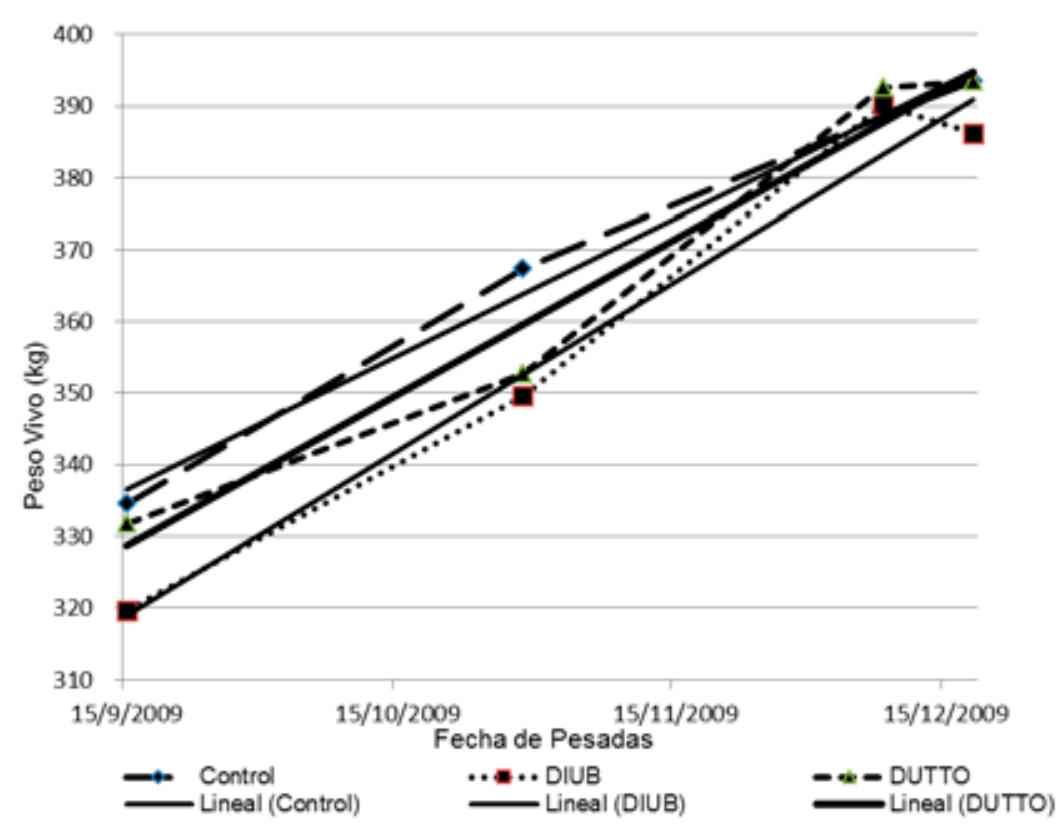

Figura 1. Promedios de peso vivo de las vacas del bloque 1 en las diferentes fechas de pesadas 
para DUTTO $y=0,8676 x-34440 \mathrm{y}$ para Control $y=0,8327 x-33037$.

En la Figura 3 se consigna el comportamiento de las pendientes para ambos bloques, agrupando los resultados de las distintas pesadas obtenidas en cada tratamiento. Las ecuaciones de regresión representadas en las líneas del gráfico fueron: para DIUB $y=25,813 x-334,75$; para DUTTO $y=24,363 x-330,96$ y para Control $y=21,701 x-343,93$.

En cuanto al análisis realizado sobre el comportamiento de los tratamientos, se pudo observar que al momento de suprimir la actividad reproductiva, el tratamiento Dutto tuvo un efecto más inmediato, suprimiendo la circulación sanguínea del ovario de una manera más efectiva en poco tiempo, teniendo la consecuencia que al ser quirúrgico genera estrés en el animal, por el dolor que produce la incisión del techo de la vagina, generando que en un principio el APV no se manifieste rápidamente. En consecuencia el efecto del tratamiento Dutto sobre el sistema reproductivo fue más inmediato y sobre el animal en general -en cuanto al APV y terminación- fue más lento pero con mejores resultados en el tiempo.

Por otro lado, el Diub no provocó un estrés marcado al ser aplicado, pero se produjo un efecto más gradual en las modificaciones reproductivas, suprimiendo la actividad sexual y generando más lentamente un efecto en el aumento de peso. La acción del Diub sobre el sistema reproductivo se manifestó más lentamente, al igual que en el APV y terminación. De todos modos, en los dos tratamientos es importante considerar la simplificación del trabajo que se puede obtener en lo que respecta al manejo de los animales, pues se trata de hembras en las que se suprime la actividad sexual.

\section{DISCUSIÓN}

En un trabajo donde los autores plantearon como objetivo medir el impacto de la edad de faena y la castración sobre la evolución del engorde, tanto en el animal en pie como en la res al gancho ${ }^{13}$, no se obtuvieron diferencias significativas atribuibles al efecto de la castración, de la edad, ni de la interacción edad por castración, resultados que coinciden con los de esta investigación.

En un estudio realizado en Brasil, donde se evaluó el APV durante las fases de amamantamiento y termi-

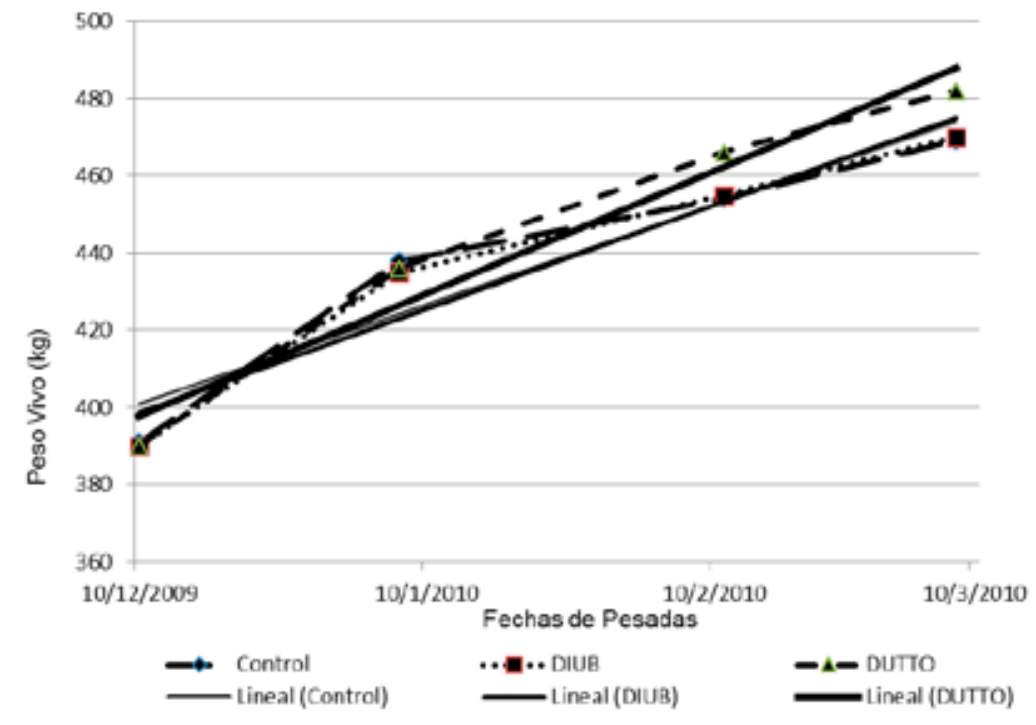

Figura 2. Promedios de peso vivo de las vacas del bloque 1 en las diferentes fechas de pesadas.

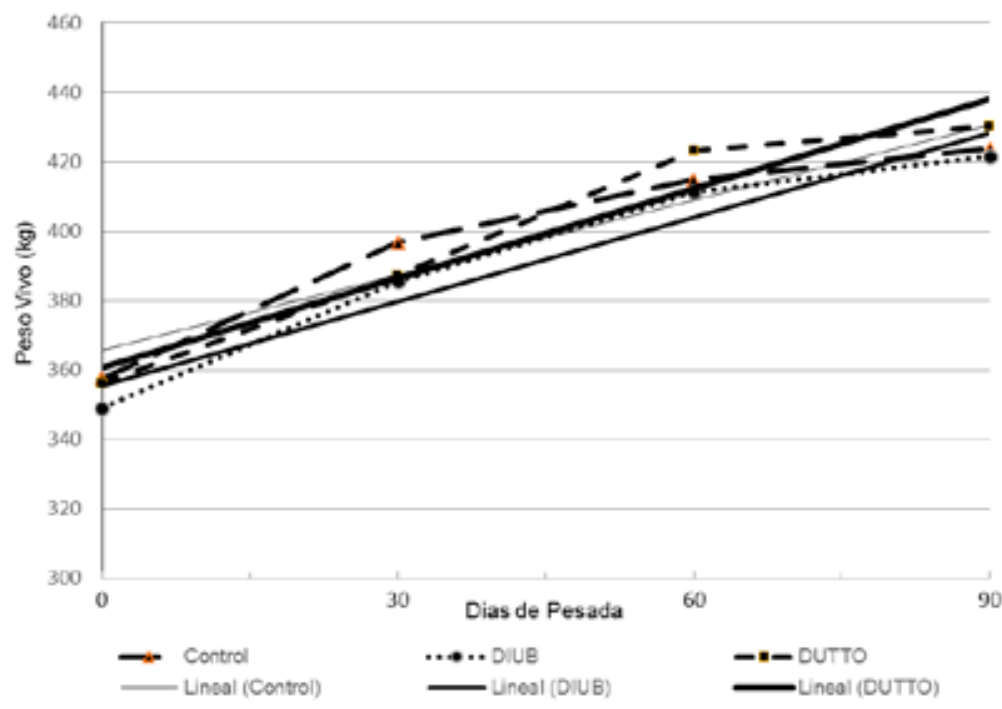

Figura 3. Promedios de peso vivo de las vacas de ambos bloques en las diferentes fechas de pesadas.

nación después del destete, de vacas castradas 45 días post-parto, se observó que durante la lactancia los terneros presentaron un APV de 276 y 280 g/día, para vacas castradas y no castradas, respectivamente ${ }^{10}$. Después del destete, en la fase de terminación de las vacas, que duró 147 días, tampoco hubo diferencias significativas en la APV entre los dos grupos (536 vs. 586 g/día), ni se observó interacción entre tratamientos y periodos.

Post-destete el desempeño de los terneros no fue afectado por el tratamiento al cual fueron sometidas las vacas. Considerando el periodo total se verificó que no hubo diferencias significativas en la ganancia de peso entre vacas no castradas y castradas, en coincidencia con el presente ensayo. A pesar de esto, los autores consideraron a la castración como un buen método para facilitar el manejo de las vacas de descarte, ya que 
pueden ser mantenidas con otras categorías, sin causar trastornos motivados por la manifestación del celo ${ }^{10}$.

En el trabajo de los creadores del Diub ${ }^{12}$, se observó que durante los primeros 60 días de estudio, el APV de las vaquillas con Diub era 16,5\% superior al grupo control. Durante los siguientes 60 días, las vaquillas tratadas con Diub tenían un promedio de APV que fue $25,5 \%$ mayor que el observado en las vaquillas control. Esto representa un aumento significativo en la tasa de crecimiento de las vaquillas con Diub entre los $61 \mathrm{y}$ 120 días.

Con el objetivo de evaluar los efectos del Diub sobre el APV, rendimiento de canal y función ovárica, analizando la ocurrencia de la androgenización natural y endógena, en una investigación se utilizaron 41 vaquillas de raza Nelore, distribuidas aleatoriamente en dos grupos experimentales (control GC y tratado GT) ${ }^{3}$. Los animales fueron mantenidos 421 días en un sistema extensivo de pastoreo con Brachiaria brizanhta cv. Marandu, suplementadas con mezcla mineral durante el período lluvioso y una mezcla proteica en el período seco. Los grupos fueron pesados cada 28 días, realizándose conjuntamente la obtención de sangre para la determinación sérica de testosterona. No hubo diferencia estadísticamente significativa entre los resultados obtenidos para GC y GT en cuanto a los parámetros de APV, PV final, rendimiento y peso de canal. Respecto a las concentraciones séricas de testosterona, tampoco fueron observadas diferencias significativas ${ }^{3}$.

En otro trabajo se evaluaron APV y GD utilizando 62 vaquillas de 14 a 16 meses de edad, las cuales fueron divididas en dos lotes, uno fue ovariectomizado (GT) por la técnica de castración transvaginal y el otro operó como lote control no ovariectomizado (GC). Al final del experimento los resultados de APV fueron mayores en GC $\left(1,332 \mathrm{~kg} /\right.$ día) que en GT $(1,118 \mathrm{~kg} / \text { día })^{7}$.

En un estudio con vaquillas mestizas Nelore $\mathrm{x}$ Angus mantenidas en pastoreo y en confinamiento, se evaluó la ganancia de PV en castradas y no castradas ${ }^{1}$. Los animales, de 18 meses de edad, fueron distribuidos en dos grupos: G1 (30 vaquillas no castradas) y G2 (30 vaquillas castradas). Todas fueron mantenidos en pastoreo de Brachiaria decumbens y pesadas a los 39 y 75 días, registrándose una diferencia significativa del APV entre los dos grupos, con $0,51 \mathrm{~kg}$ y $0,65 \mathrm{~kg}$ para G1 y G2, respectivamente. Al día 76 del ensayo, todos los animales fueron transferidos y mantenidos por 144 días en confinamiento, presentándose una diferencia significativa entre los APV de los dos grupos, con 0,73 $\mathrm{kg}$ y $0,58 \mathrm{~kg}$ para $\mathrm{G} 1$ y $\mathrm{G} 2$, respectivamente. Los resultados de este experimento permitieron demostrar que las vaquillas castradas presentaron mayor APV en pastoreo.

En otra experiencia se evaluó APV y rendimiento de la canal en 47 vacas descarte, separadas al azar en tres grupos: G1) 15 vacas controles, G2) 19 vacas castradas y G3) 15 vacas con Diub alojadas en un potrero con pastura natural ${ }^{8}$. Se pesaron al inicio y cada treinta días, durante 120 días. Al final los animales fueron faenados tras un ayuno previo de 12 horas, con el fin de determinar el rendimiento de la canal. Los resultados arrojaron diferencias de peso significativas entre G1 y G3 (120 días): 77,6 vs $108 \mathrm{~kg}$ respectivamente $(\mathrm{p}<0,05)$ y entre G2 y G3 (120 días): 81,4 vs 108 kg respectivamente $(p<0,05)$. También se encontraron diferencias significativas en el rendimiento de la canal entre G1 y G2 (51,02 vs 52,94\% respectivamente, $\mathrm{p}<0,05)$, mientras que G2 y G3 fueron similares para este parámetro. No se registraron diferencias significativas entre las ganancias de peso de G1 y G2 (120 días): 77,6 vs 81,4 kg. Los resultados demostraron que G3 fue más eficiente que G1 y G2 en ganancia de peso, en tanto que para el rendimiento de la canal G2 y G3 revelaron mejor desempeño, concluyendo que la aplicación de Diub se traduce en una mejor relación costo-beneficio ${ }^{8}$.

En un trabajo se evaluó la terminación en vientres castrados de diferente edad ${ }^{9}$, determinando el espesor de la GD y el grado de marmoreo de las reses, no encontrando efectos atribuibles a la edad $(p=0,15)$ ni la castración ( $p=0,67)$, sobre el espesor de la GD; tampoco interacción edad $\mathrm{x}$ castración $(\mathrm{p}=0,67)$. Sobre el grado de marmoreo tampoco se apreció efecto de la edad $(\mathrm{p}=0,44)$, de la castración $(\mathrm{p}=0,83)$ ni de la interacción edad x castración $(\mathrm{p}=0,68)$. Sin embargo, se observó una alta variabilidad en los datos registrados, donde los valores de espesor de la GD alcanzados (mínimo: 6,0 mm; máximo: 12,0 $\mathrm{mm}$ ), representaron un dato de indudable importancia si se consideran las elevadas velocidades de refrigeración utilizadas por la industria cárnica, que en caso de animales de baja terminación conducen a un mayor endurecimiento de la carne debido al efecto de "cold shortening". La correlación observada entre marmoreo y espesor de la grasa presentó un coeficiente $R=0,53$, no significativo $(p>0,05 ; n=25)^{9}$.

En Brasil se realizó un ensayo evaluando el efecto de la castración en vaquillas de raza Nelore destinadas a la faena ${ }^{5}$. Al final del ensayo, y luego de analizar las diferentes partes constituyentes de la carcasa de los animales castrados y no castrados, se comprobó que la castración no influenció positivamente en las características de la carcasa y de la carne, observándose que la variable área ojo de bife (“AOB”) fue la única característica que presentó diferencia significativa $(\mathrm{p}<0,05)$ entre las demás variables analizadas, siendo el valor medio para esta variable en las vaquillas no castradas $\left(54,39 \mathrm{~cm}^{2}\right)$, la cual fue $15 \%$ mayor que en las vaquillas castradas $\left(46,98 \mathrm{~cm}^{2}\right)$.

Los resultados obtenidos en las condiciones de este ensayo, permiten concluir que los métodos anticonceptivos cruentos e incruentos utilizados, no producen efectos sobre la ganancia de peso vivo ni sobre la terminación de vacas de descarte en pastoreo. De todos modos resulta interesante e importante la aplicación de los tratamientos Dutto y Diub, desde el punto de vista del manejo que puede llevarse a cabo en un establecimiento de cría donde se necesita vender gorda la hacienda de descarte, preferentemente en el segundo semestre del año, normalmente a partir del mes de junio, 
que generalmente es el momento en el cual se obtienen los mejores precios.

En todos los rodeos de cría hay toros, por lo cual existe el riesgo de que se presenten preñeces no deseadas, por tal motivo al incorporar una de estas técnicas se deberá suprimir la actividad reproductiva de los animales, permitiendo que las vacas tratadas compartan potreros con cualquier categoría (toros, novillos), facilitando el manejo para la utilización de potreros con mejor oferta forrajera. Ello evitaría que cuando llegue el momento de venta de ese vientre para faena, se encuentre preñada $o$ a punto de parir.

Desde otro punto de vista, también puede destacarse que en animales con importante desgaste de los incisivos y una alimentación a campo natural con deficiencias en cuanto a la calidad de la pastura, es probable que no se observen diferencias en el aumento de peso, y esto quizá se deba al efecto mecánico negativo del desgaste y de la dieta mencionada, lo cual imposibilita la expresión positiva de los cambios metabólicos inducidos por los tratamientos en vacas viejas.

Por tal motivo y a pesar de que otros autores no encontraron diferencias significativas en vaquillas castradas sometidas a confinamiento ${ }^{1}$, sería importante analizar el comportamiento de vacas viejas de descarte en establecimientos de engorde a corral (feed-lot). En este sistema, donde los animales reciben una dieta equilibrada de buena calidad y se suprime el pastoreo directo, se minimizaría el efecto del estado de dentición deteriorado de vacas viejas comparado con las vaquillas, lo cual podría ser motivo de próximas investigaciones.

Agradecimientos. Al establecimiento Yatay Corá y al Dr. Arnaldo Ragazzi, veterinario a cargo. Al INTA EEA Corrientes y personal del grupo de producción animal. Al Dr. Stahringer y resto del equipo de reproducción de la EEA INTA Colonia Benítez, por las determinaciones ecográficas.

\section{REFERENCIAS}

1. Chacur M, De Faria M, Oba E, Kronka S. 2007 Efeito da ovariectomia em novilhas Nelore $\mathrm{x}$ Angus, Bos taurus indicus $\mathrm{x}$ Bos taurus taurus sobre os ganhos de peso. $\mathrm{Ci}$ ênc Agr Londr 28: 317-322.
2. Cruzate GA, Panigatti JL, Moscatelli G. 2011. Mapa descriptivo de suelos y ambientes de la Provincia de Corrientes. INTA. http://inta.gob.ar/imagenes/Corrientes. jpg/view (acceso: 15/4/14).

3. Da Silva H, Gambarini M, Oliveira B, Gordo J. 2005. Efeitos da utilização do dispositivo intra-uterino (DIU) sobre o ganho de peso e o rendimento de carcaça de novilhas nelore destinadas ao abate. Ciênc Anim Bras 6: 95-103.

4. Dutto L. 1981. La castración de vacas, Ed. AgropecuariaHemisferio Sur, Montevideo, 205 p.

5. Franco L, Pinheiro A, Prado C, Soares M, Pádua J, Miyagi E, Gonçalves K, Machado M, Barbosa V. 2007. Características de carcaça e carne em novilhas castradas ou não-castradas da raça Nelore. Ciênc Anim Bras 8: 777785.

6. Infostat. 2010. Versión 1.1. Grupo Infostat, Facultad de Ciencias Agropecuarias, Universidad Nacional de Córdoba, Argentina.

7. Meirelles C, Bueno C, Ernandes L, Weiss R, Saporski M. 2007. Avaliação do ganho de peso de novilhas ovariectomizadas por técnica transvaginal. Ciênc Agr Amb (Curitiba) 5: 303-307.

8. Orquera M, Pochon D, Flores S, Konrad J, Crudeli G. 2011. Rendimiento de la canal y ganancia de peso en vacas de descarte con inducción del anestro por vías quirúrgica versus mecánica. Rev Vet 22: 64-67.

9. Pasinato A, Monje A, Vittone S, Galli I, Teira G, Tisocco O, Perlo F, Bonato P. 2005. Pautas para la certificación de calidad de carnes en vacas. 4. Marmoreo y espesor de la grasa. Congr Arg Prod Anim (P.28), Bahía Blanca, Argentina.

10. Restle J, Grassi C. 1993. Castração de vacas de descarte e seu efeito no ganho de peso da vaca e do bezerro. Pesq Agrop Bras 28: 1437-1441.

11. Stahringer R, Chifflet S. 2006. Cartilla descriptiva: grado de condición corporal en vacas de cría y frame score (escala de tamaño). www.braford.com.ar

12. Turín E, Nagle C, Lahoz M, Torres M, Turín M, Mendizabal AF, Escofet M. 1997. Effects of a coffer-bearing intrauteeine device on the ovarian function, body weight gain and pregnancy rate of nulliparous heifers. Theriogenology 47: 1327-1336.

13. Vittone S, Monje A, Teira G, Perlo F, Bonato P, Pasinato A, Galli I. 2005. Pautas para la certificación de calidad de carnes en vacas. Memorias 28 Congreso Argentino de Producción Animal, Bahía Blanca, Argentina. 